\title{
Research on the Sample Size Calculation Method of the Inertial Gyro Based on the Step-stress Accelerated Life-test
}

\author{
Jinxin XU \& Jinzhu WANG \& Zhifeng FAN \\ Ordnance Engineering College, Shijiazhuang, China
}

\begin{abstract}
It is difficult to verify the reliability of a new type of ammunition components through traditional statistical testing methods. And the accelerated life test of ammunition components at the present often requires a large amount of sample size. The calculation model for minimum sample size of the Inertial Gyro has been established by clearing the accelerated life test method of Inertial Gyro, considering the Weibull distribution is assumed to product life, and taking advantage of a priori information. And the minimum sample size of the Inertial Gyro that step-stress accelerated life-test required has been calculated out.
\end{abstract}

KEYWORD: Inertial Gyro, Accelerated life test, Weibull distribution, Minimum sample size.

\section{INTRODUCTION}

The Inertial Gyro of a type of guided projectile is mechatronic product, it has a high requirement for the precision and storage reliability of the inertial gyro. The experimental data of the natural storage test is the most actual and accurate. But it takes too long times, so it is not suitable for the development of new ammunition. Therefore, we propose to use accelerated life test method-In a relatively short period of time, The reliability and failure distribution of the normal stress level are deduced, and predicted the storage life.

At present, the accelerated life test method for ammunition components is mainly about the "ammunition components accelerated life test method" (GJB 5103-2004). However, due to the test sample size is too large. The minimum sample size of the inertial gyro is the high-precision and electromechanical. The equipment is limit and expensive, so the sample size is often unable to meet the requirements of the test.

In this paper, we have adequate to use of the effective prior information, and researches on the minimum test sample size calculation of the inertial gyro accelerated life test.

\section{ACCELERATED LIFE TEST}

Accelerated life testing of guided ammunition components which means that in a short test period time and maintain a constant failure mechanism. We increased the stress of the test to shorten the test period. Then get the normal storage environment of the qualitative variation rule.

According to the test of stress application methods, Accelerated life test can be divided into three types: Constant stress accelerated life test, Step-stress accelerated life-test and Progressive stress accelerated life test. We have adopted a constant relative humidity stress, step temperature stress accelerated life test method in gyro accelerated life test. This is based on the following two considerations:

1) The inertial gyro is sealed and packaged in the factory. Their packing is made of metal, which is sealed by the roller mouth and so on. Through theoretical analysis and engineering test we found the packing cylinder only through temperature but not wet. Therefore, when we constant acceleration test of inertial gyro. We can constant relative humidity stress and increasing temperature stress.

2) If we used constant stress accelerated life test with two factors of temperature and humidity. The testing process is not only simple, but also the data processing is not particularly complex. The biggest drawback is that the test time is too long. The step stress accelerated life test can eliminate the mistake, which is the step can enhance with time. The method is to divide a certain number of samples into several groups, each groups set up a time of increasing stress level. In the test, the stress level is according to the prescribed time increased from the low to the high level, as shown in Figure 1.This test sample can be used in a relatively short period of time to 
experience the whole process of life and the test time is greatly reduced. The statistical analysis of the sample failure information to make the test time greatly reduced.

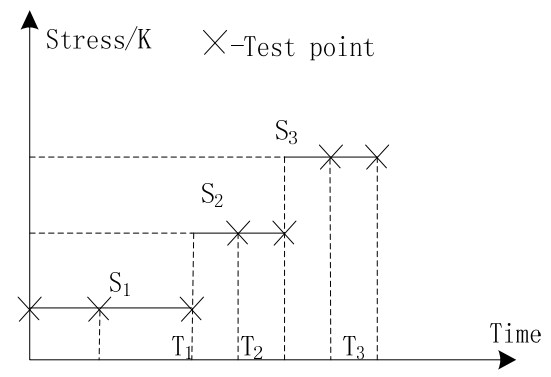

Figure 1 Schematic diagram of accelerated life test

\section{SAMPLE SIZE CALCULATION}

\subsection{Determination principle}

Due to the Inertial Gyro is currently less equipped, and the price is more expensive. So when determining the sample size, a priori information should be used to reduce the sample size as much as possible. In order to ensure the success of the test, and accurate prediction of its reliable storage life, to determine the sample should follow the following principles:

a. At the end of each stress level test, the number of newly added failure samples is not less than 1;

b. The confidence level is not less than 0.8 when deriving the lower limit of reliability according to the average reliability.

c. Sample size as little as possible

\subsection{A priori information of Storage reliability distribution}

According to the research achievements for decades of the Ordnance Technology Research Institute of the General Armament Department, the variation law of ammunition storage reliability with storage time can be described by Weibull distribution. With the difference of the storage conditions, distribution parameters were difference, but generally do not affect the form of distribution function. So we considered that the variation law of ammunition storage reliability with storage time following Weibull distribution,

$$
R(t)=e^{-(t / \eta)^{m}}
$$

It can be concluded that average storage reliability at normal temperature is:

$$
R_{S, T}(t)=e^{-\left(t / \eta_{T}\right)^{m}}
$$

The lower limit of storage reliability at normal temperature is:
$R_{S, L}(t)=e^{-\left(t / \eta_{L}\right)^{m}}$

Where " $\mathrm{t}$ " is the storage time to the factory; " $\mathrm{m}$ " is the shape parameter; " $\eta_{T}$ " is the average characteristic life; " $\eta_{L}$ " is the lower limit characteristic life, and according to the formula (2) (3):

$$
\begin{aligned}
& \eta_{T}=T_{R L} /\left(-I n R_{T}\right)^{1 / m} \\
& \eta_{L}=T_{R L} /\left(-I n R_{L}\right)^{1 / m}
\end{aligned}
$$

Where " $T_{R L}$ " is the reliable storage life for lower limit of reliability of 0.9 and confidence level of 0.8 , " $R_{L}$ " is the lower limit of reliability, " $R_{T}$ " is the average reliability which is corresponding to the lower limit of reliability.

\subsection{Minimum sample size estimation}

According to the literature, it can be considered that the distribution of the reliability valuation " $R(t)$ " is a normal approximation distribution which the mean is " $R_{T}(t)$ " and the covariance is " $D\left[R_{T}(t)\right]$ ". According to normal approximation method,

$$
\begin{gathered}
D\left[R_{T}(t)\right]=\left[R_{T}(t) \operatorname{InR}_{T}(t)\right]^{2}\{1.109 \\
-0.514 \operatorname{In}\left[-\operatorname{InR}_{T}(t)\right] \\
\left.+0.608\left(\operatorname{In}\left[-\operatorname{InR}_{T}(t)\right]\right)^{2}\right\} / n
\end{gathered}
$$

(6)When " $\mu_{\gamma}$ " is noted to the upper quantile of the standard normal distribution, the lower limit of reliability for the confidence level of 0.8 is:

$$
\begin{gathered}
R_{L}(t)=R_{T}(t)-\mu_{\gamma} \bullet\left(D\left[R_{T}(t)\right]\right)^{1 / 2} \\
\text { If: } \\
\begin{aligned}
y(x)=[x \operatorname{In} x]^{2}\{1.109-0.514 \operatorname{In}[-\operatorname{In} x] \\
\left.+0.608(\operatorname{In}[-\operatorname{In} x])^{2}\right\} \\
(0<x<1)
\end{aligned}
\end{gathered}
$$

According to formula (6) (7), to ensure the confidence level reach to " $\gamma$ " when deriving " $R_{L}(t)$ "according to " $R_{T}(t)$ ", then the lower limit of sample size is:

$[n]=\frac{\mu_{\gamma}{ }^{2}}{\left[R_{T}(t)-R_{L}(t)\right]^{2}} \bullet y\left[R_{T}(t)\right]$

That is, $[\mathrm{n}]$ is the minimum sample size to meet the confidence level.

The failure number of minimum requirements for 1 , the lower limit of sample size when the lower limit of reliability is " $R_{L}(t)$ " is:

$$
[n]_{1}=\frac{1}{1-R_{L}(t)}
$$

So, the sample size can be determined if it's meet the needs of both [n] and [n] . 


\section{EXAMPLE}

Now take a number of inertial gyro has been stored for 14 years, through the relevant technical conditions and calculation, we know that $R_{L}=0.913, R_{T}=0.953, \quad m=1.059 T_{R L}=10.25$, $\eta_{T}=179.8, \eta_{L}=98.54$. Calculating " $R_{T}(t)$ " and " $R_{L}(t)$ " according to formula (2) and (3), the relevant calculation results were listed as follows when $\gamma=0.8\left(\mu_{\gamma}=0.80329\right)$ (as shown in Table 1$)$.
According to the calculation results, finally we got the initial sample size $\mathrm{n}_{1}=8$ in order to reduce the sample size as much as possible. That means the initial acceleration test stress " $\mathrm{T}_{1}$ " and acceleration time " $\tau 1$ " should be able to guarantee the equivalent storage time of " $\tau$ ef, 1 " (Cumulative value of the ratio of storage time and average characteristic life span under different stress levels) is greater than 0.1494 . Equivalent to 26.9 years of storage at room temperature effect.

Table 1 Minimum sample size estimation(1)

\begin{tabular}{cccccccccc}
\hline $\mathrm{t}$ & 0.0 & 4.5 & 10.25 & 15.0 & 20.0 & 30.0 & 40.0 & 50.0 & 60.0 \\
\hline$\tau_{\mathrm{ef}}{ }^{2}$ & 0.0 & 0.02503 & 0.05701 & 0.08343 & 0.0112 & 0.1668 & 0.2225 & 0.2781 & 0.3337 \\
$\mathrm{R}_{\mathrm{T}}(\mathrm{t})$ & 1.0 & 0.9801 & 0.9530 & 0.9305 & 0.9069 & 0.8606 & 0.8158 & 0.7727 & 0.7314 \\
$\mathrm{y}\left[\mathrm{R}_{\mathrm{T}}(\mathrm{t})\right]$ & & 0.00481 & 0.01739 & 0.02996 & 0.04393 & 0.07128 & 0.09566 & 0.1160 & 0.1323 \\
$\mathrm{R}_{\mathrm{L}}(\mathrm{t})$ & 1.0 & 0.9626 & 0.9130 & 0.8726 & 0.8313 & 0.7529 & 0.6805 & 0.6142 & 0.5536 \\
$\mathrm{R}_{\mathrm{T}}(\mathrm{t})-\mathrm{R}_{\mathrm{L}}(\mathrm{t})$ & 0.0 & 0.01745 & 0.0400 & 0.05785 & 0.07558 & 0.1077 & 0.1253 & 0.1585 & 0.1778 \\
{$[\mathrm{n}]$} & & 10.20 & 7.02 & 5.78 & 4.96 & 3.96 & 3.93 & 2.98 & 2.70 \\
{$[\mathrm{n}]_{1}$} & & 26.74 & 11.49 & 7.85 & 5.93 & 4.05 & 3.13 & 2.59 & 2.24 \\
\hline
\end{tabular}

Notes: (1) Confidence level is 0.8 , Minimum failure number is 1 ;

(2) $\tau$ ef $=\mathrm{t} / \eta \mathrm{T} 0, \quad \eta \mathrm{T} 0=179.8 \mathrm{y}$.

\section{CONCLUSION}

In this article, By clearing the accelerated life test method of Inertial Gyro, and based on taking advantage of a priori information, and considering the Weibull distribution is assumed to product life, the calculation model for minimum sample size of the Inertial Gyro has been established. And the minimum sample size of the Inertial Gyro that stepstress accelerated life-test required has been calculated out. And reference is providing for the sample size calculation method for accelerated life testing of similar products. With the continuous upgrading of new ammunition products, researching of product reliability will continue to develop. Therefore, it is more and more important to determine the accelerated life test sample of the inertial gyro, which is a kind of expensive component.

\section{REFERENCE:}

Donghua ZHAO, Huaizhi ZHANG, Shenggiang GUO.et al. Prediction of Terminal Guided Projectile Storage Life Based on Gray Model[J]. Equipment Environmental Engineering, 2011,8(6):28-30.

Minglun LI, Dongyang LI, Bo ZHENG. Ammunition Storage Reliability[M]. Beijing: National Defend Industry Press, 1997:35-53.

Ordnance Engineering College. 1999. Data Processing Report of DRD13 Fuze Accelerated Life Test.

Ordnance Technology Research Institute. 1990. Test Report on Typical Ammunition Storage Reliability.
Shisong MAO, Lingling WANG. 1997. Accelerated Life Test. Shanghai: Science Press.

Shanghai University. 1999. Data Processing Report of Ammunition Components Accelerated Life Test. 